\title{
Efeito da crioterapia e termoterapia associados ao alongamento estático na flexibilidade dos músculos isquiotibiais
}

\author{
Effects of cryotherapy and thermotherapy associated with static \\ stretching on the flexibility of hamstring muscles
}

\author{
S.A. Silva, D.J. Oliveira, M.J.N. Jaques, R.C. Araújo
}

\begin{abstract}
RESUMO
O objetivo do presente estudo foi analisar o efeito da crioterapia e do aquecimento muscular sobre a flexibilidade dos isquiotibiais associados com 3 minutos de alongamento estático. Para isso, 40 jovens voluntários, de ambos os sexos, foram distribuídos de forma aleatória em quatro grupos: Grupo 1 Controle, Grupo 2 - Alongamento estático mantido por 3 minutos, Grupo 3 - Alongamento e aquecimento por 20 minutos e Grupo 4 - Alongamento e crioterapia por 20 minutos. Foram realizadas três series de alongamento durante cinco dias consecutivos, sendo realizadas avaliações do ângulo extensor do joelho ao início do protocolo, depois de cada dia e ao final do protocolo. Para a comparação intergrupos foi utilizada a ANOVA com post-hoc Tukey e para a análise intragrupo foi utilizado o teste t pareado, todos com nível de significância de $5 \%$. Os resultados demonstraram que todos os grupos submetidos ao alongamento apresentaram ganho de flexibilidade em relação ao grupo controle. No entanto, não houve diferença estatística entre os grupos submetidos aos diferentes programas de alongamento. Conclui-se que o aumento da flexibilidade se deve ao alongamento e independe da aplicação prévia de hipertermia e/ou hipotermia.
\end{abstract}

Palavras-chave: crioterapia, termoterapia, alongamento, flexibilidade

ABSTRACT

The objective of the present study was to analyze the effect of cryotherapy and muscular warming on the flexibility of the hamstring muscles associated with three minutes of static stretching. Forty young male and female volunteers were randomly included in one of four groups: Group 1 - Control group, Group 2 - Three minutes of static stretching, Group 3 - Stretching preceded by warming using shortwave diathermy (20 minutes), and Group 4 - Stretching preceded by applying cryotherapy (20 minutes) to the posterior thigh region. The program consisted of three series of stretching during five consecutive days and flexibility was assessed by goniometric evaluations of the extensor angle of the knee at the beginning of the protocol, at the end of the day and at the end of the protocol. The intergroup comparison was made through ANOVA post-hoc Tukey and the intragroup by paired t test, all with 5\% level of significance. The three experimental groups significantly increased their range of motion in relation to the control group. However, differences were not observed among groups submitted to the different stretching programs. In conclusion, increases in flexibility were due to stretching and did not depend on previous application of hyperthermia and/or hypothermia. Keywords: cryotherapy, termotherapy, stretching, flexibility

Submetido: 29.03.2010 | Aceite: 30.05.2010

Simone Alves da Silva, Denílson José de Oliveira e Márcio José Nunes Jaques. Graduação de Fisioterapia da Universidade de Pernambuco - UPE, Campus Petrolina, Brasil.

Rodrigo Cappato de Araújo. Departamento de Fisioterapia da Universidade de Pernambuco - UPE, Campus Petrolina, Brasil.

Endereço para correspondência: Rodrigo Cappato de Araújo, Universidade de Pernambuco - Campus Petrolina, BR 203 Km 2, s/n, Vila Eduardo, CEP 56.300-000, Petrolina - PE, Brasil.

E-mail: rodrigo.cappato@upe.br 
A flexibilidade recebe inúmeras definições, sendo a mais utilizada, a simples habilidade de movimentar uma articulação através da amplitude de movimento (ADM) disponível, livre de dor, restrições e sem provocar stresse no sistema músculo-tendídeo (Carregaro, Silva, \& Gil Coury, 2007). Os factores que afectam a flexibilidade são vários, entre os quais, o sedentarismo, a imobilidade prolongada, a prática de actividades repetitivas e especialmente no caso dos músculos isquiotibiais, a permanência prolongada na postura sentada. Estes factores podem levar a um encurtamento dos músculos, afectando sua capacidade de deformação e podendo causar alterações na mecânica dos membros inferiores e coluna vertebral. Além de aumentar o risco de desenvolvimento de disfunções posturais e predisposição de lesões do aparelho locomotor (Davis, Ashby, McCale, McQuain, \& Wine, 2005; Decoster, Scanlon, Horn, \& Cleland, 2004; Herbert \& Xavier, 2003).

Com o objectivo de proporcionar a manutenção e/ou o aumento da flexibilidade, muitos profissionais tem utilizado diferentes técnicas de alongamento muscular, sendo a mais utilizada, o alongamento estático (Viveiros, Polito, Simão, \& Farinatti, 2004).

O alongamento estático é uma técnica que consiste em alongar passivamente um segmento à máxima amplitude possível, utilizando força manual ou mecânica e mantendo-o por um período específico de tempo. Esse tipo de exercício seria responsável pela redução da resistência muscular, devido ao aumento da viscoelasticidade da unidade motora, resultando no aumento do comprimento muscular. Segundo diferentes autores, esses factores poderiam prevenir lesões, desde que o alongamento seja realizado de forma crónica antes e após programas de exercício (Almeida et al., 2009; Shrier \& Gossal, 2000; Viveiros et al., 2004).

Entretanto, não há um consenso de como executá-lo e garantir uma máxima eficácia, além de sugerir dúvidas com relação ao número de séries, frequência e duração dos programas de alongamento. Algumas pesquisas têm descrito o uso de recursos térmicos associados a estes programas com o objectivo de optimizar os resultados (Branco et al., 2006; Signori, Voloski, Kerkhoff, Brig, \& Plentz, 2008; Viveiros et al., 2004).

A associação dos recursos térmicos aos programas de alongamento tem sido utilizada com o intuito de proporcionar maiores ganhos de amplitude de movimento. No entanto, não existe consenso na literatura a respeito de protocolos específicos para o uso de tais recursos associados ao exercício de alongamento. Taylor, Waring e Brashear (1995) avaliaram os ganhos de flexibilidade dos músculos isquiotibiais em voluntários submetidos a protocolos de alongamento estático realizado isoladamente e combinado com o uso de calor e frio. Os resultados demonstraram que o alongamento, quando associado ao uso de calor, proporcionou maior ganho de flexibilidade, quando comparado ao uso da técnica realizada de forma isolada e quando associada ao frio.

Dessa forma, o maior ganho proporcionado pela associação do calor, seria explicado pelo aumento $\mathrm{da}$ extensibilidade das fibras colágenas, diminuição da viscosidade e tensão tecidual. Isso, favoreceria o relaxamento das propriedades mecânicas do músculo, diminuindo, assim, o espasmo muscular e o stiffness articular (Draper, Knight, Fujiwara, \& Castel, 1999; Draper, Miner, Knight, \& Ricard, 2002; Funk, Swank, Adams, \& Treolo, 2001; O’Sullivan, Muray, \& Sainsbury, 2009).

De forma distinta, Cornelius e Jackson (1984) observaram que os ganhos de flexibilidade muscular foram maiores no grupo que associou o alongamento por facilitação neuromuscular proprioceptiva ao uso da crioterapia. Apesar do pouco conhecimento e entendimento na literatura do modo pelo qual a crioterapia associada ao alongamento poderia optimizar os ganhos de flexibilidade, alguns pesquisadores embasados nos reconhecidos efeitos fisiológicos da aplicação do frio, têm sugerido mecanismos que poderiam explicar 
estes resultados, tais como, diminuição da velocidade de condução nervosa, do reflexo de estiramento e analgesia local (Kanlayanaphotporn \& Janwantanakul, 2005; Knight, 2000; Swenson, Sward, \& Karlsson, 1996).

Esses efeitos permitiriam maior amplitude de movimento durante a realização dos exercícios de alongamento, devido à menor sensação de desconforto e dor durante a execução do alongamento. Sendo um factor muito importante, pois geralmente observa-se que a limitação álgica durante as manobras de alongamento antecede a limitação tecidual, o que isso poderia comprometer a eficiência do exercício (Brasileiro, Faria, \& Queiroz, 2007).

Estudos mais recentes (Draper et al., 2002; Funk et al., 2001) demonstraram que a associação de recursos térmicos ao exercício de alongamento não oferece maiores ganhos de flexibilidade quando comparado à realização do alongamento feito de forma isolada.

Contudo, observa-se ampla divergência metodológica em todos os estudos, sendo eles, realizados em diferentes amostras, com diversas técnicas de alongamento e variados protocolos de aplicação dos recursos termoterapêuticos. Portanto, não se encontra na literatura um consenso em relação à eficiência da associação entre os programas de alongamento e o uso de recursos térmicos e principalmente, quais seriam as técnicas mais apropriadas para promover aumento de flexibilidade. O objectivo deste estudo foi verificar a influência dos recursos termoterapêuticos quando associados a exercícios de alongamento estático mantido por três minutos com intensidade constante, durante um período curto de treinamento.

\section{MÉTODO}

\section{Amostra}

$\mathrm{O}$ estudo foi realizado em quarenta indivíduos jovens de ambos os sexos com média de idades de $21.97 \pm 3.28$ anos, todos sedentários, sem história de lesão musculoesquelética e/ou cirurgia em membros inferiores e que apresentaram amplitude de extensão do joelho inferior ou igual a $160^{\circ}$ graus. Os indivíduos que praticavam alguma actividade física há pelo menos seis meses, e que possuíam hipersensibilidade à aplicação de gelo ou calor, foram excluídos do estudo em questão. Todos os indivíduos leram e assinaram um termo de consentimento, segundo a resolução n. ${ }^{\circ}$ 196/96 do Conselho Nacional de Saúde. Além disso, este estudo foi submetido e aprovado pelo Comité de Ética da Universidade de Pernambuco-UPE.

\section{Procedimentos}

Inicialmente, foi realizada uma avaliação física composta por uma anamnese e um exame físico, durante os quais foram colhidos dados pessoais, antropométricos e avaliado o ângulo de extensão activa do joelho direito dos voluntários. Para a colecta da extensão activa do joelho (EAJ) direito, o participante foi colocado em uma maca, em decúbito dorsal, tendo o quadril direito flectido a $90^{\circ}$ e o membro inferior esquerdo mantido em extensão na maca. Este posicionamento foi mantido por dois pesquisadores, enquanto foi solicitado ao voluntário estender activamente o joelho direito. Um terceiro pesquisador realizou então a goniometria. Para isso, foi utilizado um goniómetro da marca $\mathrm{CARCI}^{\circledR}$ (São Paulo, Brasil), sendo este posicionado da seguinte maneira: o eixo foi colocado no côndilo lateral, o braço fixo direccionado para o trocânter maior do fémur e o braço móvel em paralelo à perna. As medidas foram realizadas três vezes consecutivas e posteriormente foi feito uma média aritmética.

Posteriormente, os indivíduos foram distribuídos de forma aleatória em quatro grupos com dez componentes:

Grupo 1 - Grupo controle, ou seja, os indivíduos pertencentes a esse grupo não foram submetidos aos exercícios de alongamento, apenas foram realizadas as avaliações inicial e final, antes e após o período de estudo.

Grupo 2 - Os indivíduos foram submetidos a um protocolo de uma série de alongamento 
estático dos músculos isquiotibiais mantidos por três minutos, realizado por cinco dias consecutivos.

Grupo 3 - Os indivíduos foram submetidos ao mesmo procedimento do grupo 2. No entanto, os exercícios de alongamento foram precedidos de aplicação calor por meio do equipamento de ondas curtas modelo Diatermed (CARCI ${ }^{\circledR}$ - São Paulo, Brasil), com disposição coplanar das placas na região posterior da coxa por vinte minutos, no modo contínuo e intensidade de $80 \mathrm{~W}$ (Brasileiro et al., 2007). Optou-se por este protocolo de diatermia por ondas curtas, pois estudos anteriores demonstraram que quando aplicado em intensidades altas, o mesmo é capaz de produzir um aquecimento tecidual na ordem de 4.0 a $4.6^{\circ} \mathrm{C}$ em grandes áreas e com uma profundidade de até três centímetros (Brown \& Baker, 1987; Draper et al., 1999; Robertson, Ward, \& Jung, 2005).

Grupo 4 - Os indivíduos foram também submetidos ao procedimento de alongamento estático, porém, precedido pela aplicação de crioterapia. Para isso, os voluntários foram posicionados em decúbito ventral, em seguida a região posterior da coxa foi coberta por uma bandagem para evitar ulcerações provocadas pelo frio e uma bolsa de gel resfriada foi colocada sobre a musculatura citada. Além disso, foi aplicada compressão na região por meio de bandagem elástica. $\mathrm{O}$ tempo de aplicação da técnica de crioterapia foi de vinte minutos.

As medidas de amplitude articular foram realizadas antes e após cada sessão de alongamento para avaliar os efeitos imediatos da intervenção, enquanto as medidas que foram feitas antes e após o término dos protocolos do programa foram utilizadas para avaliar os efeitos em médio prazo. O programa constou de cinco sessões de alongamento, sendo que a ultima avaliação goniométrica foi realizada setenta e duas horas após o término do programa.

Os procedimentos de alongamento estático foram realizados sobre uma maca, com o voluntário em decúbito dorsal. O membro inferior esquerdo permaneceu fixo sobre a maca, sendo estabilizado por um dos pesquisadores. $\mathrm{O}$ membro inferior direito foi posicionado com o joelho totalmente estendido e quadril em flexão. Por meio de um sistema de polia e corda, uma extremidade foi fixada em torno do tornozelo direito e outra extremidade com um peso de sete quilos. Este peso realizava a flexão passiva do quadril até à amplitude máxima suportada por cada voluntário, mantendo desta forma uma intensidade constante do exercício de alongamento durante três minutos.

\section{Análise Estatística}

As análises estatísticas foram realizadas no programa SPSS, versão 11.0. Antes da análise de cada variável, a normalidade na distribuição dos dados foi verificada por meio do teste de Kolmogorov-Smirnov. Para a análise do efeito agudo, os valores de amplitude de movimento de extensão do joelho foram aferidos antes e após cada sessão. $\mathrm{Na}$ análise do efeito crónico, foram registados os ângulos pré e póstreinamento. As diferenças entre as medidas foram avaliadas por meio de análise de variância (ANOVA), com medidas repetidas com post-hoc de Tukey. Para comparação entre os valores pré-treinamento e pós-treinamento intragrupo foi utilizado o teste $t$ pareado. Em todas as situações foi utilizado um nível de significância de $p<.05$.

\section{RESULTADOS}

São apresentados na figura 1 , os valores de média e desvios-padrão encontrados antes do programa de alongamento e após seu término, de cada grupo, relacionados ao teste de flexibilidade dos músculos isquiotibiais. Através da análise estatística observou-se que todos os grupos apresentaram aumento da amplitude articular $(p<.05)$, excepto o grupo controle. 


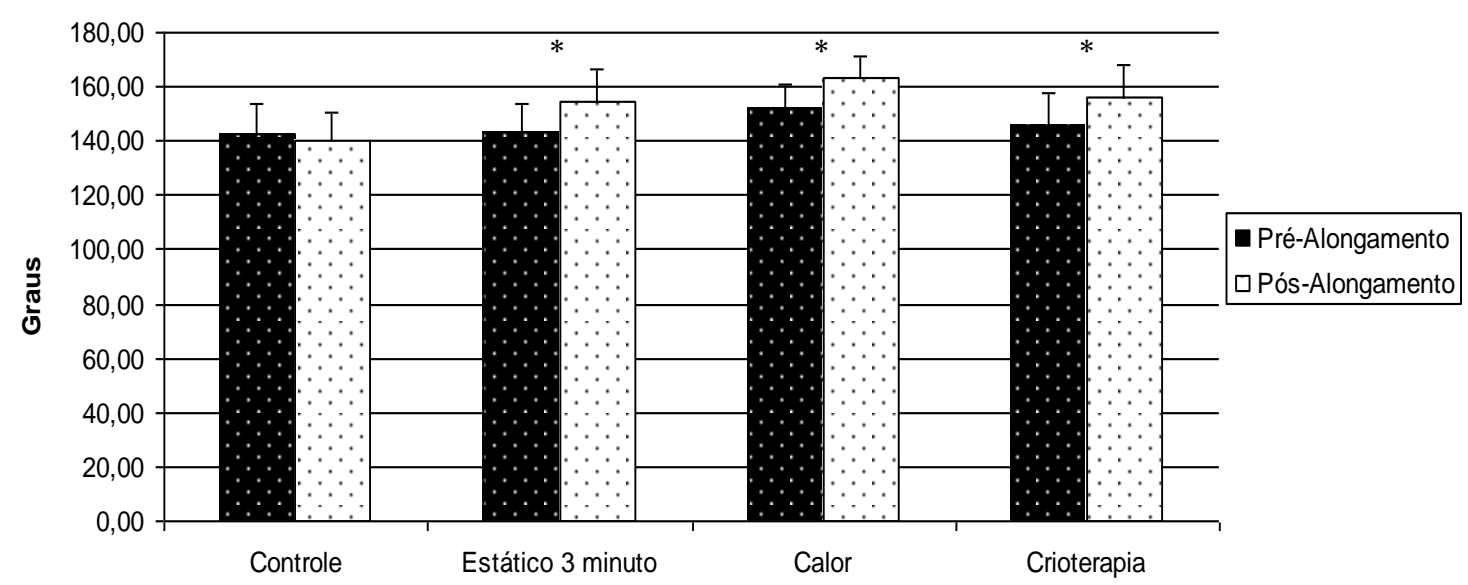

Figura 1. Valores de amplitude articular (graus) antes e após o programa de alongamento; ${ }^{*} p<.05$

As médias do ganho final de amplitude articular foram: grupo controle: $-1.87^{\circ}$, grupo alongamento 3 minutos estático: $11.87^{\circ}$, grupo calor: $11.40^{\circ}$ e grupo crioterapia: $9.87^{\circ}$.

Por meio da análise estatística (ver figura 2) observou-se diferença significativa entre os grupos submetidos aos programas de alongamento em relação ao grupo controle ( $p$ $<$.05). No entanto, não houve diferença significativa entre os grupos submetidos às diferentes técnicas $(p>.05)$.

Por outro lado, a análise dos valores médios de ADM de cada grupo submetido aos diferentes programas de alongamento (ver figura 3) demonstrou que o grupo 3 (calor) apresentou ganho significativo a partir da segunda avaliação $(p<.05)$, enquanto o grupo crioterapia apresentou ganho a partir da terceira avaliação $(p<.05)$ e 0 grupo alongamento estático, somente apresentou diferença entre a primeira e a ultima avaliação $(p<.05)$.

\section{DISCUSSÃO}

O presente estudo objectivou avaliar a influência do uso de recursos termoterapêuticos em associação com a técnica de alongamento estático, no ganho de flexibilidade da musculatura posterior da coxa durante um curto período de treinamento.

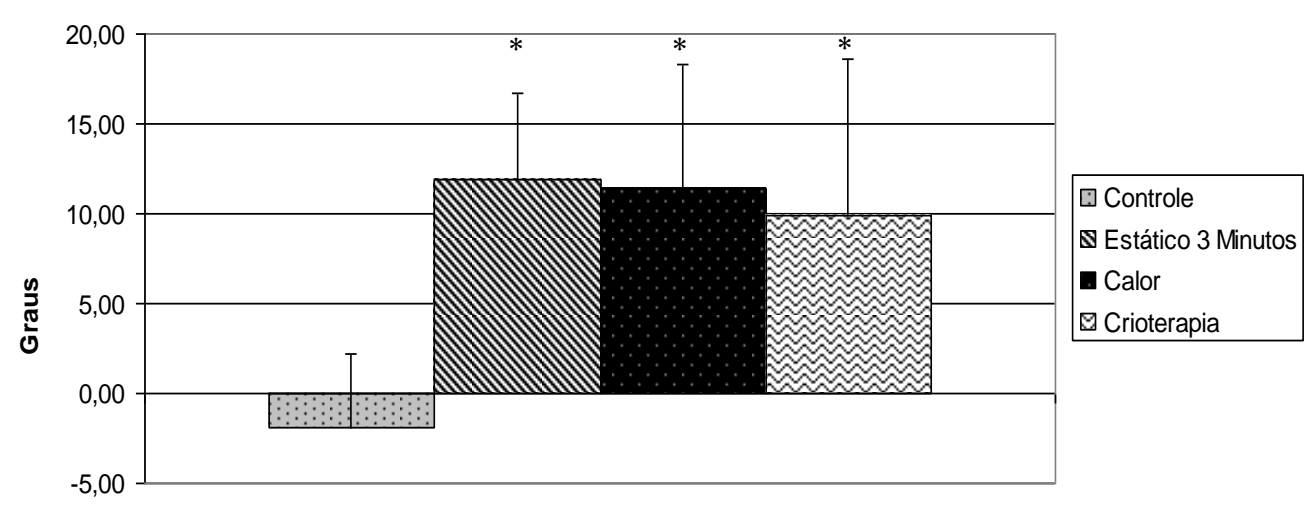

Figura 2. Médias dos ganhos de amplitude articular após programa de alongamento $* p<.05-$ Diferença em relação ao grupo controle 


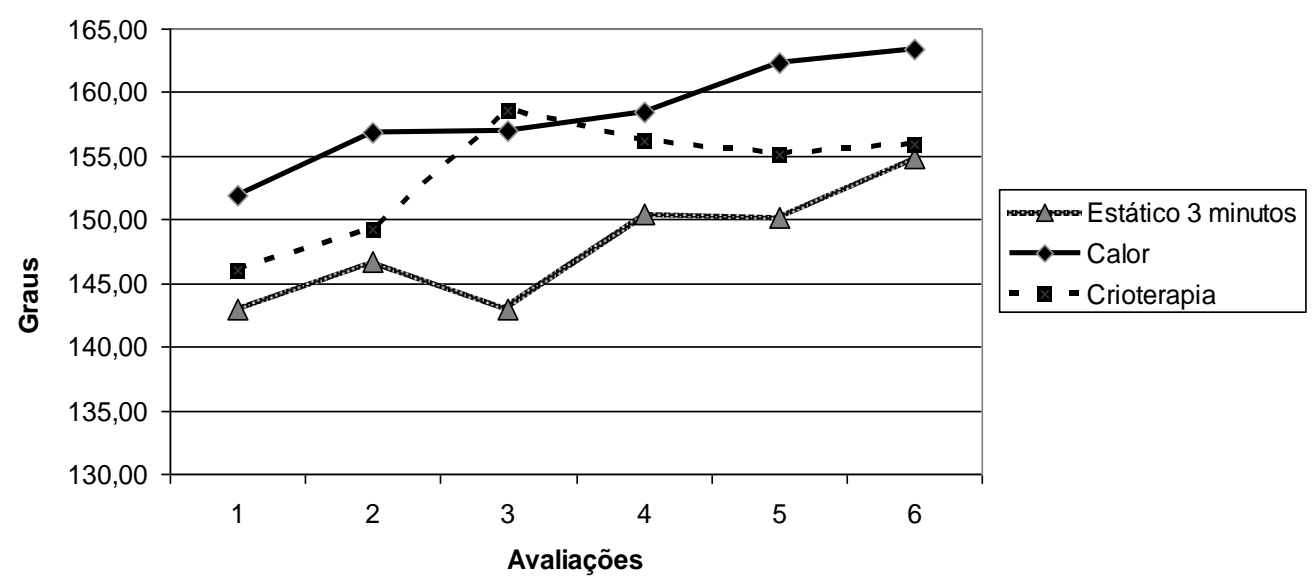

Figura 3. Valores de ADM ao longo de todo o programa de alongamento

De fato, foi possível confirmar que o exercício de alongamento é eficaz em propiciar ganho de flexibilidade, mesmo quando executado em um curto período de tempo. Por outro lado, a aplicação prévia de recursos termoterapêuticos não influenciou no aumento de amplitude de movimento ao final do programa.

Ao se analisar o valor de amplitude de movimento ao longo de cada sessão observouse, a partir da segunda avaliação, uma maior efectividade no grupo submetido à associação do alongamento precedido da aplicação de calor, sugerindo que o uso da diatermia por ondas curtas foi eficaz para o aumento da temperatura local, corroborando com vários autores (Draper et al., 1999; Lentell, Hetherington, Eagan, \& Morgan, 1992; Robertson et al., 2005) que descrevem aumento da temperatura muscular na ordem de $4{ }^{\circ} \mathrm{C}$ em uma profundidade de três a cinco centímetros e que perduraram no mínimo cinco minutos.

Este aumento da temperatura local proporcionaria um aumento da extensibilidade das fibras colágenas, alterando as propriedades viscoelásticas e mecânicas do tecido muscular, facilitando o ganho de flexibilidade (Draper, Castro, Feland, Schulthies, \& Eggett, 2004; Peres, Draper, Knight, \& Ricard, 2002).
De acordo com esta teoria, o uso da crioterapia poderia proporcionar um impacto negativo no ganho de flexibilidade, uma vez que se observa na literatura que o resfriamento dos tecidos seria responsável pelo aumento da rigidez do tecido conjuntivo e diminuição da extensibilidade (Knight, 2000). No entanto, no presente estudo pôde-se observar que a aplicação do frio também proporcionou ganhos significativos de ADM nas primeiras sessões.

Este ganho pode ser explicado por dois factores, o primeiro estaria relacionado ao fato da hipotermia causar uma diminuição do espasmo muscular e do reflexo de estiramento, por meio da redução do input sensorial do fuso muscular que é responsável por uma facilitação do estímulo motor e aumento da tensão do músculo (Eston \& Peres, 1999). Outro factor positivo à crioterapia seria a diminuição da sensibilidade dolorosa ao alongamento por meio da redução da velocidade de condução nervosa (Proulx, Ducharme, \& Kenny, 2003). Uma vez reduzida esta sensação, maior seria a tolerância do voluntário a manobra, permitindo um alongamento mais efectivo devido à diminuição da limitação álgica que frequentemente precede a limitação mecânica tecidual (Brasileiro et al., 2007).

O grupo de voluntários submetidos somente ao alongamento apresentou ganhos 
similares de flexibilidade em relação aos demais grupos que fizeram uso dos recursos térmicos, confirmando que o aumento da flexibilidade está directamente relacionado ao estímulo mecânico, que seria responsável pelo remodelamento dos tecidos conjuntivo e musculotendíneo. A manutenção do alongamento promoveria aumento não só da extensibilidade, mas também no comprimento muscular e nas estruturas das proteínas contráteis dos sarcômeros (Prado et al., 2005).

$O$ ganho de flexibilidade do grupo submetido ao alongamento, somente foi evidenciado ao final do programa, sugerindo que o alongamento estático mantido por três minutos é eficaz para o ganho de flexibilidade se for realizado por no mínimo cinco sessões. Além disso, os recursos térmicos parecem facilitar o ganho no início do programa, possivelmente por minimizar as limitações teciduais e álgicas presentes durante a manobra de alongamento. No entanto, esta a relação de ganhos obtidos no início não foi mantida nas últimas sessões do programa, apresentando resultados similares ao programa de alongamentos realizados de forma isolada.

Portanto, a aplicação prévia de recursos térmicos ao alongamento estático não confere maiores ganhos de flexibilidade em indivíduos saudáveis. Fato este, que torna dispensável a associação prévia de tais recursos, pois os ganhos imediatos obtidos pela associação do alongamento com o calor e o frio não foram mantidos até o final do programa, sugerindo que o sucesso do programa aplicado a sujeitos saudáveis está relacionado à eficiência do exercício de alongamento.

Os resultados sugerem que na prática clínica, não seria uma boa estratégia de tratamento, associar recursos termoterapêuticos ao alongamento muscular, com o objectivo de acelerar ou aumentar os ganhos de flexibilidade, pelo menos em indivíduos sedentários sem histórico de lesões.

No entanto, mais estudos devem ser realizados, sobretudo com o objectivo de avaliar os efeitos da associação dos recursos térmicos e diferentes técnicas de alongamento em indivíduos que apresentem diminuição da ADM causada por alterações musculoarticulares e que estejam em processo de reabilitação, assim como em atletas. Para que se possa então, justificar ou não, o uso de tais recursos, que aumentam consideravelmente a complexidade e o tempo de atendimento, e consequentemente aumentam as despesas dos serviços de reabilitação.

\section{CONCLUSÕES}

A partir dos resultados encontrados no presente estudo, conclui-se que o alongamento estático proporciona aumento da flexibilidade de músculos encurtados independentemente do uso de recursos térmicos, mesmo em um curto período de tempo. No entanto, em futuros estudos se deve avaliar protocolos mais longos, bem como, a associação dos recursos térmicos com outras técnicas de alongamento em diferentes grupos musculares.

\section{REFERÊNCIAS}

Almeida, P. H. F., Barandalize, D., Ribas, D. I. R., Gallon, D., Macedo, A. C. B., \& Gomes, A. R. S. (2009). Alongamento muscular: Suas implicações na performance e na prevenção de lesões. Revista Fisioterapia em Movimento, 22(3), 335-343.

Branco, V. R., Negrão Filho R. F., Padovani, C. R., Azevedo, F. M., Alves, N., \& Carvalho, A. C. (2006). Relação entre a tensão aplicada e a sensação de desconforto nos músculos isquiotibiais durante o alongamento. Revista Brasileira de Fisioterapia, 10(4), 465-472.

Brasileiro, J. S., Faria, A. F., \& Queiroz, L. L. (2007). Influência do resfriamento e do aquecimento local na flexibilidade dos músculos isquiotibiais. Revista Brasileira de Fisioterapia, $11(1), 57-61$.

Brown, M., \& Baker, R. D. (1987). Effect of pulsed short wave diathermy on skeletal muscle injury in rabbits. Physical Therapy, 67, 208-214.

Carregaro, R. L., Silva, L. C. C., \& Gil Coury, H. J. C. (2007). Comparação entre dois testes clínicos para avaliar a flexibilidade dos músculos posteriores da coxa. Revista Brasileira de Fisioterapia, 11 (2), 139-145. 
Cornelius, W., \& Jackson, A. (1984). The effects of cryotherapy and PNF on hip extensor flexibility. Journal of Athletic Training 19, 183199.

Davis, D. S., Ashby, P. E., McCale, K. L., McQuain, J. A., \& Wine, J. M. (2005). The effectiveness of 3 stretching techniques on hamstring flexibility using consistent stretching parameters. Journal of Strength and Conditioning Research, 19(1), 27-32.

Decoster, L. C., Scanlon, R. L., Horn, K. D., \& Cleland, J. (2004). Standing and supine hamstring stretching are equally effective. Journal of Athletic Training, 9(4), 330-334.

Draper, D., Knight, K., Fujiwara, T., \& Castel, C. (1999). Temperature change in human muscle during and after pulsed short-wave diathermy. Journal of Orthopaedic \& Sports Physical Therapy, 29(1), 13-18.

Draper, D. O., Castro, J. L., Feland, B., Schulthies, S., \& Eggett, D. (2004). Shortwave diathermy and prolonged stretching increase hamstring flexibility more than prolonged stretching alone. Journal of Orthopaedic \& Sports Physical Therapy, 34(1), 13-20.

Draper, D. O., Miner, L., Knight, K. L. \& Ricard, M. D. (2002) The carry-over effects of diathermy and stretching in developing hamstring flexibility. Journal of Athletic Training, 37(1), 3742.

Eston, R., \& Peres, D. (1999). Effects of cold-water immersion on the symptoms of exerciseinduced muscle injury. Journal of Sports Science, 17(3), 231-238.

Funk, D. C., Swank, A. M., Adams, K. J., \& Treolo, D. (2001). Efficacy of moist heat pack application over static stretching on hamstring flexibility. Journal of Strength and Conditioning Research, 15(1), 123-126.

Herbert, S., \& Xavier, R. (2003). Ortopedia e traumatologia: Princípios e práticas. Porto Alegre: Artmed.

Kanlayanaphotporn, R., \& Janwantanakul, P. (2005). Comparison of skin surface temperature during the application of various cryotherapy modalities. Archives of Physical Medicine and Rehabilitation, 85, 1411-1415.

Knight, K. L. (2000). Crioterapia no tratamento das lesões desportivas. São Paulo: Manole.
Lentell, G., Hetherington, T., Eagan, J., \& Morgan, M. (1992). The use of thermal agents to influence the effectiveness of a low-load prolonged stretch. Journal of Orthopaedic \& Sports Physical Therapy, 16(5), 200-207.

O'Sullivan, K., Muray, E., \& Sainsbury, D. (2009). The effect of warm-up, static stretching and dynamic stretching on hamstring flexibility in previously injured subjects. BMC Musculoskeletal Disorders, 10(37), 1-9.

Peres, S. E., Draper, D. O., Knight, K. L., \& Ricard, M. D. (2002). Pulsed shortwave diathermy and prolonged long-duration stretching increase dorsiflexion range of motion more than identical stretching without diathermy. Journal of Athletic Training, 37(1), 43-50.

Prado, L. G., Makarenko, I., Andresen, C., Kruger, M., Opitz, C. A., \& Linke, W. A. (2005). Isoform diversity of giant proteins in relation to passive and active contractile properties of rabbit skeletal muscles. Journal of General Physiology, 126(5), 461-480.

Proulx, C. I., Ducharme, M. B., \& Kenny, G. P. (2003). Effect of water temperature on cooling efficiency during hyperthermia in humans. Journal of Applied Physiology, 94(4), 1317-1323.

Robertson, V. J., Ward, A. R., \& Jung, P. (2005). The effect of heat on tissue extensibility: A comparison of deep and superficial heating. Archives of Physical Medicine and Rehabilitation, 86(8), 19-25.

Shrier, L., \& Gossal, K. (2000). Myths and truths of stretching. The Physician and Sportsmedicine, 28(8), 57-63.

Signori, L. U., Voloski, F. R. S., Kerkhoff, A. C., Brig, L., \& Plentz, R. D. M. (2008). Efeito de agentes térmicos aplicados previamente a um programa de alongamentos na flexibilidade dos músculos isquiotibiais encurtados. Revista Brasileira de Medicina do Esporte, 14(4), 328-333.

Swenson, C., Sward, L., \& Karlsson, J. (1996). Cryotherapy in sports medicine. Scandinavian Journal of Medicine \& Science in Sports, 6, 193-200.

Viveiros, L., Polito, M. D., Simão, R., \& Farinatti, P. (2004). Respostas agudas imediatas e tardias da flexibilidade na extensão do ombro em relação ao número de séries e duração do alongamento. Revista Brasileira de Medicina do Esporte, 10(6), 459-463. 